\title{
Zeno Subspaces for Coupled Superconducting Qubits
}

\author{
Paolo Facchi, ${ }^{1,2}$ Rosario Fazio, ${ }^{3,4}$ Giuseppe Florio, ${ }^{2,5}$ \\ Saverio Pascazio, ${ }^{2,5}$ and Tetsuya Yoneda ${ }^{5,6}$
}

Received June 17, 2005 / Published online April 4, 2006

Decoherence is one of the most serious drawback in quantum mechanical applications. We discuss the effects of noise in superconducting devices (Josephson junctions) and suggest a decoherence-control strategy based on the quantum Zeno effect.

KEY WORDS: decoherence; quantum Zeno effect, Josephson junctions.

PACS: 03.67.Pp; 03.65.Xp.

\section{INTRODUCTION}

This paper is dedicated to Prof. Emilio Santos on the occasion of his 70th birthday. His numerous contributions in the foundation of quantum mechanics range from stochastic electrodynamics and optics to the Bell inequality, subjects that were considered controversial until a couple of decades ago. We therefore find it appropriate to celebrate Emilio's birthday by discussing a novel issue of practical interest that stems from the foundation of quantum physics.

Quantum mechanics is a relatively young theory and was originally conceived in order to describe the behavior of microscopic systems, such as atoms and molecules. It is an extremely successful theory and hinges upon the superposition principle, a basic postulate according to which the

\footnotetext{
${ }^{1}$ Dipartimento di Matematica, Università di Bari, I-70126 Bari, Italy.

${ }^{2}$ INFN, Sezione di Bari, I-70126 Bari, Italy.

${ }^{3}$ NEST-INFM \& Scuola Normale Superiore, Piazza dei Cavalieri 7, 56126 Pisa, Italy.

${ }^{4}$ International School for Advanced Studies (SISSA) via Beirut 2-4, I-34014 Trieste, Italy.

${ }^{5}$ Dipartimento di Fisica, Università di Bari, I-70126 Bari, Italy. E-mail: saverio.pascazio@ ba.infn.it

${ }^{6}$ School of Medical Sciences, Kumamoto University, 4-24-1 Kuhonji, 862-0976 Kumamoto, Japan.
} 
linear combination of two or more quantum states is a possible state. The validity of this principle for the solutions of the equations of motion requires these equations to be linear. Both are essential ingredients of the quantum theory and are profoundly related to each other.

The superposition principle is experimentally very well confirmed for microscopic systems. However, the last few decades have witnessed a growing interest towards the existence of quantum superpositions of larger objects. Such systems can be mesoscopic or even macroscopic and the application of quantum mechanical concepts and principles therein has important consequences. Among the most interesting systems of this kind there are Josephson junctions and superconducting quantum interference devices.

One of the most detrimental effects against the coherent features of these superconducting devices is decoherence ${ }^{(1)}$. Decoherence tends to suppress quantum superpositions, making these systems "classical". (2) We will consider the general problem of decoherence in Josephson systems, with particular reference to the question of the preservation of quantum superpositions. ${ }^{(3)}$ Potential sources of decoherence are phonons, (normal) electrons, nuclear spins, the electromagnetic field and the measurement system (performing the read-out of the quantum state and enabling de facto a reliable inference of the degree of quantum mechanical coherence). All these sources of decoherence can be considered as being part of the "environment" surrounding the superconducting device. We will focus in particular on the implications for practical quantum computation. ${ }^{(4)}$

\section{THE MODEL}

Let us consider a Josephson junction, in the configuration shown in Fig. 1. Its Hamiltonian in the charge regime reads

$$
H_{\mathrm{J}}=\frac{\varepsilon}{2} \sigma_{z}-\frac{E_{J}}{2} \sigma_{x}, \quad \varepsilon\left(V_{\mathrm{g}}\right)=4 E_{\mathrm{C}}\left(1-C_{\mathrm{g}} V_{\mathrm{g}} / e\right),
$$

where $E_{\mathrm{C}}=e^{2} / 2\left(C_{\mathrm{g}}+C_{\mathrm{J}}\right)$ is the charging energy, assumed to be much larger than $E_{\mathrm{J}}$ (Josephson coupling), and $\sigma_{i}$ 's $(i=1,2,3)$ are the Pauli matrices.

The Hamiltonian of the junction in its environment is written as

$$
H=H_{\mathrm{J}}+\eta(t) H_{\mathrm{dec}},
$$

where the action of the environment is modeled by the noise term $\eta H_{\mathrm{dec}}$, $\eta$ being a Gaussian stochastic process with

$$
\langle\eta(t)\rangle=0, \quad\left\langle\eta(t) \eta\left(t^{\prime}\right)\right\rangle=F_{\tau_{0}}\left(t-t^{\prime}\right),
$$




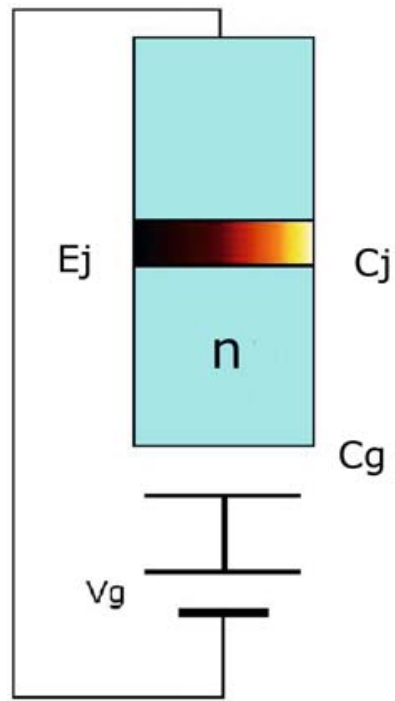

Fig. 1. A Josephson junction: $n$ is the number of excess Cooper pairs, $V_{\mathrm{g}}$ the gate potential, $C_{\mathrm{g}}$ and $C_{\mathrm{J}}$ the gate capacitance and the tunnel capacitance, respectively. $E_{\mathrm{J}}$ is the Josephson coupling. The superconducting regions, in light blue, enclose the junction.

the brackets denoting the average over all the possible realizations of the noise. $F_{\tau_{0}}$ is a generic function vanishing at $\pm \infty$, whose width $\tau_{0}$ is the correlation time of the environment.

It is well known that there is no general recipe in order to get in a rigorous way the "noise" terms from the total Hamiltonian (describing the environment + the system). As a matter of fact, this program can be carried out only in some particular cases, ${ }^{(5)}$ that have played an important historical role in clarifying the features of quantum dissipative phenomena. The more pragmatic approach to the dynamics of two-level systems, based on Eq. (2.2), has been used, e.g., in Ref. [6].

\section{DECOHERENCE}

For sufficiently small values of $\tau_{0}$, the density matrix $\rho(t)$ of the quantum system approximately satisfies the equation $(\hbar=1)$

$$
\begin{aligned}
\dot{\rho}(t)= & -i\left[H_{\mathrm{J}}, \rho(t)\right]-\int_{0}^{t} d s F_{\tau_{0}}(s) \\
& e^{-i H_{\mathrm{J}} s}\left[H_{\mathrm{dec}}^{I}(s),\left[H_{\mathrm{dec}}, \rho(t-s)\right]\right] e^{i H_{\mathrm{J}} s},
\end{aligned}
$$


where $H_{\mathrm{dec}}^{I}(s)=e^{i H_{\mathrm{J}} t} H_{\mathrm{dec}} e^{-i H_{J} t}$ is the interaction picture of $H_{\mathrm{dec}}$. If the correlation time $\tau_{0}$ is much smaller than all other characteristic times of the problem, one can use the white-noise approximation

$$
F_{\tau_{0}}(t)=\tau_{0} \delta(t)
$$

and obtain a Gorini-Kossakowski-Sudarshan-Lindblad master equation. ${ }^{(7,8)}$

$$
\begin{aligned}
\dot{\rho}(t) & =-i\left[H_{\mathrm{J}}, \rho(t)\right]-\frac{\tau_{0}}{2}\left[H_{\mathrm{dec}},\left[H_{\mathrm{dec}}, \rho(t)\right]\right] \\
& =-i\left[H_{\mathbf{J}}, \rho(t)\right]-\frac{\tau_{0}}{2}\left\{H_{\mathrm{dec}}^{2}, \rho(t)\right\}+\tau_{0} H_{\mathrm{dec}} \rho(t) H_{\mathrm{dec}},
\end{aligned}
$$

where $\{\cdot, \cdot\}$ is the anticommutator.

In general, in the two-level approximation, the operator $H_{\text {dec }}$ in (2.2) can be expressed as a superposition of the Pauli operators and must be chosen according to the physical features of the system investigated. In the following we will take

$$
H_{\mathrm{dec}}=\Delta \sigma_{z}
$$

in order to model the effects of charge noise. In this case, from Eq. (3.3), the decoherence/dissipation rate is clearly of order $\Delta^{2} \tau_{0}$.

The noise provokes in general a relaxation towards a mixed state, deteriorating the quantum features of the system (decoherence). If one deals with a two-level system, like that in Fig. 1, there is no interesting way to preserve the purity of the quantum state: indeed, assume that we prepare the junction in a superposition of "up" $(n=1)$ and "down" $(n=0)$ states. A possible mechanism for preventing the deteriorating effects of noise is to hinder any motion, "freezing" de facto the system in one of the (only) two available states. This is clearly not interesting, e.g. in the context of quantum computation, where state manipulation is necessary. There is, however, one possible way out, that consists in enlarging the size of the Hilbert space and constraining the motion in a multidimensional subspace. Physically, this can be achieved by coupling two (or more) Josephson devices and will be discussed in the following.

\section{COUPLING OF JOSEPHSON JUNCTIONS}

In general, the Hamiltonian describing two interacting Josephson systems can be written in the form:

$$
H=H_{0}+K H_{\text {int }}=H^{(1)}+H^{(2)}+K H_{\text {int }},
$$


where $H_{0}=H^{(1)}+H^{(2)}$ is the total Hamiltonian of the uncoupled junctions, $H^{(1)}$ and $H^{(2)}$ have the structure (2.2) and the interaction $H_{\text {int }}$ depends on the physical features of the coupling. We shall focus on the most common coupling mechanisms and consider the interaction via: (a) a capacitance, (b) another Josephson junction, and (c) an inductance. The interaction Hamiltonians read

$$
\begin{aligned}
H_{\text {int }}^{\text {capacitance }} & \propto \sigma_{z}^{(1)} \sigma_{z}^{(2)}, \\
H_{\text {int }}^{\text {Josephson }} & \propto \sigma_{+}^{(1)} \sigma_{-}^{(2)}+h . c ., \\
H_{\text {int }}^{\text {inductance }} & \propto \sigma_{y}^{(1)} \sigma_{y}^{(2)},
\end{aligned}
$$

and the devices are sketched in Figs. 2, 3 and 4, respectively. We will see that one can counter decoherence by increasing the coupling $K$ in (4.1). The idea hinges upon the quantum Zeno effect $^{(9)}$ and quantum Zeno subspaces. ${ }^{(10)}$

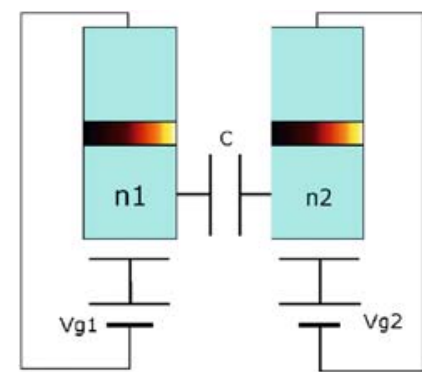

Fig. 2. Josephson junctions coupled via a capacitance $C$.

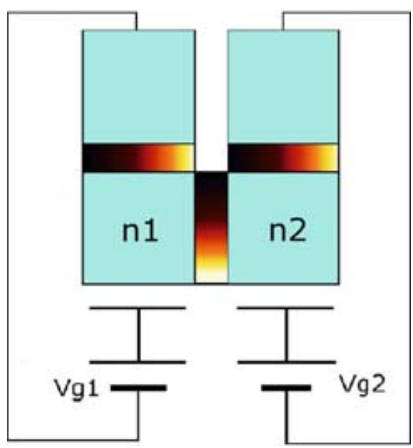

Fig. 3. Josephson junctions coupled via a third Josephson junction. 


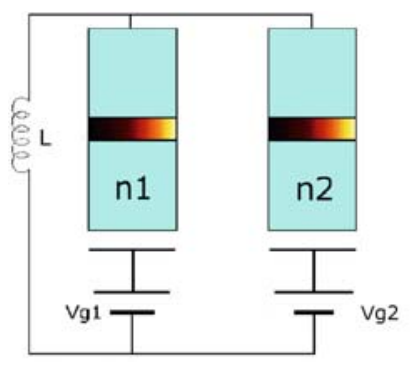

Fig. 4. Josephson junctions coupled via an inductance $L$.

\section{QUANTUM ZENO SUBSPACES}

Consider a system described by the Hamiltonian

$$
H_{K}=H_{0}+K H_{\text {int }},
$$

where $H_{0}$ is the uncontrolled Hamiltonian and $K$ defines the strength of the interaction. Let

$$
H_{\text {int }} P_{n}=\lambda_{n} P_{n},
$$

so that $P_{n}$ is the orthogonal projection onto $\mathcal{H}_{P_{n}}$, the eigenspace of $H_{\text {int }}$ belonging to the eigenvalue $\lambda_{n}$. In the large- $K$ limit the generator of the evolution becomes: ${ }^{(10)}$

$$
\begin{aligned}
H_{K} \stackrel{K \longrightarrow \infty}{\longrightarrow} H_{\text {diag }} & =\hat{P} H_{K}=\sum_{n} P_{n} H_{K} P_{n} \\
& =\sum_{n}\left(P_{n} H_{0} P_{n}+K \lambda_{n} P_{n}\right) \\
& =H_{\mathrm{Z}}+K H_{\mathrm{int}}=H_{\mathrm{diag}}
\end{aligned}
$$

It is important to notice that in Eq. (5.3) the eigenvalues are in general distinct, $\lambda_{n} \neq \lambda_{m}$ for $n \neq m$, and the $\mathcal{H}_{P_{n}}$ 's are in general multidimensional. The limiting evolution operator has the explicit form

$$
U_{K}(t)=\exp \left(-i H_{K} t\right) \stackrel{K \longrightarrow \infty}{\longrightarrow} \mathcal{U}(t)=\exp \left(-i H_{\text {diag }} t\right),
$$

so that in the large- $K$ limit also the evolution operator becomes diagonal with respect to $H_{\text {int }}$ :

$$
\left[\mathcal{U}(t), P_{n}\right]=0 .
$$


In words, in the $K \rightarrow \infty$ limit an effective superselection rule arises and the total Hilbert space is split into (Zeno) subspaces $\mathcal{H}_{P_{n}}$ that are invariant under the evolution. The dynamics within each subspace $\mathcal{H}_{P_{n}}$ is governed by the diagonal part $H_{\mathrm{Z}} P_{n}=P_{n} H_{0} P_{n}$ of the free Hamiltonian $H_{0}$ [plus a (sector-dependent) superselection charge $K \lambda_{n} P_{n}$ ]. We stress that the superselection rules discussed here are a consequence of the Zeno dynamics (strong coupling) and are equivalent to the celebrated " $\mathrm{W}^{3}$ " ones. ${ }^{(11)}$ The total Hilbert space splits into Zeno subspaces which do not communicate. If decoherence is suppressed within some (or even only one) of them, one obtains decoherence-free subspaces ${ }^{(12)}$ via a Zeno mechanism (strong continuous coupling).

For example, consider a Hamiltonian of the type (2.2), involving noise terms that are responsible for decoherence: in order to obtain a decoherence-free subspace (whose projector is $P_{n}$ ) it is sufficient to require the minimal condition

$$
P_{n} H_{\mathrm{dec}} P_{n}=\alpha_{n} P_{n} .
$$

The evolution of the density matrix within the subspace $\mathcal{H}_{P_{n}}$ is unitary, as one can easily see from (5.3)-(5.4). It is important to notice that in order to encode a qubit the dimension of this subspace should be $\operatorname{dim}\left(\mathcal{H}_{P_{n}}\right) \geqslant 2$ [hence the simple system described by (2.2) does not suffice].

In practice the control of decoherence becomes effective when

$$
K \gg 1 / \tau_{0},
$$

Where $\tau_{0}$ is the correlation time of the environment, introduced in Eq. (2.3). From this condition we realize that this kind of control is useless in the (idealized) case of white noise (for which the correlation time is strictly 0 ). Physically, this simply means that the correlation time of the noise is the shortest timescale of the problem and it is impossible to devise a control mechanism (that should make use of strong couplings whose inverse strength $1 / K \ll \tau_{0}$, according to the preceding equation).

\section{EXAMPLE OF COUPLED JOSEPHSON QUBITS}

Let us consider two coupled Josephson qubits described by the Hamiltonian (4.1):

$$
H=H^{(1)}+H^{(2)}+K H_{\text {int }},
$$

where the coupling $H_{\text {int }}$ can be of any of the forms (4.2)-(4.4). Let the basis of the 4-dimensional Hilbert space be 


$$
\begin{aligned}
& |0\rangle=|00\rangle=\left(\begin{array}{l}
0 \\
1
\end{array}\right) \otimes\left(\begin{array}{l}
0 \\
1
\end{array}\right)=\left(\begin{array}{l}
0 \\
0 \\
0 \\
1
\end{array}\right), \\
& |1\rangle=|01\rangle=\left(\begin{array}{l}
0 \\
1
\end{array}\right) \otimes\left(\begin{array}{l}
1 \\
0
\end{array}\right)=\left(\begin{array}{l}
0 \\
0 \\
1 \\
0
\end{array}\right), \\
& |2\rangle=|10\rangle=\left(\begin{array}{l}
1 \\
0
\end{array}\right) \otimes\left(\begin{array}{l}
0 \\
1
\end{array}\right)=\left(\begin{array}{l}
0 \\
1 \\
0 \\
0
\end{array}\right), \\
& |3\rangle=|11\rangle=\left(\begin{array}{l}
1 \\
0
\end{array}\right) \otimes\left(\begin{array}{l}
1 \\
0
\end{array}\right)=\left(\begin{array}{l}
1 \\
0 \\
0 \\
0
\end{array}\right) .
\end{aligned}
$$

The eigenvalues and eigenprojectors generated by the couplings (4.2)-(4.4) are easily calculated.

(a) Capacitance (4.2):

$$
\left\{\begin{array}{l}
P_{1}=\{|0\rangle,|3\rangle\}, \quad \lambda_{1}=+1 \\
P_{-1}=\{|1\rangle,|2\rangle\}, \quad \lambda_{-1}=-1
\end{array}\right.
$$

In this case we have two 2-dimensional subspaces in which we can in principle encode a qubit.

(b) Josephson junction (4.3):

$$
\begin{cases}P_{0}=\{|0\rangle,|3\rangle\}, & \lambda_{0}=0 \\ P_{ \pm}=\left\{\frac{|1\rangle+|2\rangle}{\sqrt{2}}\right\}, & \lambda_{ \pm}= \pm 1\end{cases}
$$

We get one 2-dimensional subspace and two 1-dimensional subspaces. A qubit can in principle be encoded in the 2-dimensional subspace. 
(c) Inductance (4.4):

$$
\left\{\begin{array}{cc}
P_{1}=\{|++\rangle,|--\rangle\}, & \lambda_{1}=+1 \\
P_{-1}=\{|+-\rangle,|-+\rangle\}, & \lambda_{-1}=-1
\end{array}\right.
$$

with

$$
\begin{gathered}
| \pm \pm\rangle=\frac{|0\rangle-|3\rangle \mp i(|2\rangle+|1\rangle)}{2}=\frac{1}{2}\left(\begin{array}{c}
-1 \\
\mp i \\
\mp i \\
1
\end{array}\right), \\
|\mp \pm\rangle=\frac{|0\rangle+|3\rangle \mp i(|1\rangle-|2\rangle)}{2}=\frac{1}{2}\left(\begin{array}{c}
1 \\
\pm i \\
\pm i \\
1
\end{array}\right) .
\end{gathered}
$$

We again obtain two 2-dimensional spaces, in which we can in principle encode a qubit.

We now assume that (6.1) contains a charge noise Hamiltonian of the form

$$
H_{\mathrm{dec}}(t)=\eta_{1} H_{\mathrm{dec}}^{(1)}+\eta_{2} H_{\mathrm{dec}}^{(2)}=\eta_{1} \Delta \sigma_{z}^{(1)}+\eta_{2} \Delta \sigma_{z}^{(2)},
$$

where $\eta_{i}(i=1,2)$ are two independent Gaussian stochastic processes with the property (2.3). Let us evaluate the possibility of controlling the effects of $H_{\mathrm{dec}}$ in the strong coupling limit. We easily realize that the deteriorating effects of this kind of noise can be reduced only in the case of the "inductive coupling" (4.4), (6.8), because in such a case

$$
P_{1} H_{\mathrm{dec}} P_{1}=P_{-1} H_{\mathrm{dec}} P_{-1}=0,
$$

so that Eq. (5.6) is satisfied with $\alpha_{1}=\alpha_{-1}=0$. In this case, the strong coupling splits the total Hilbert space into two 2-dimensional subspaces $\mathcal{H}_{P_{1}}, \mathcal{H}_{P_{-1}}$, between which the noise is unable to provoke transitions: each subspace becomes decoherence free (see Fig. 5).

In the other two cases Eq. (6.12) does not hold (or is valid just for a 1-dimensional subspace where one cannot encode a qubit) and the subspaces are useless for storing information. 


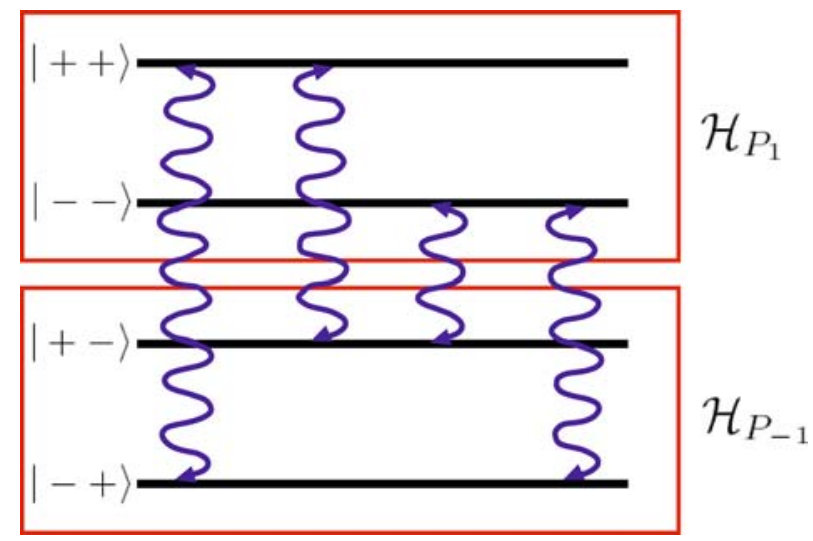

Fig. 5. Strong inductive coupling (4.4). The projections [red rectangular boxes, see Eq. (6.8)] split the total Hilbert space into two subspaces $\mathcal{H}_{P_{1}}, \mathcal{H}_{P_{-1}}$. Noise-induced transitions (wavy lines) are suppressed by the strong coupling and each subspace becomes decoherence free.

\section{DIFFERENT KINDS OF NOISE AND FINAL REMARKS}

In a Josephson device the noise can have several expressions, according to the operating regime and the physical setup. If the noise is mainly due to electrostatic charge effects, Eq. (6.11) is a good approximation. In general, if one assumes that there is a "dominating" source of noise in each Josephson device, the part of the Hamiltonian that is responsible for decoherence can be written as

$$
H_{\mathrm{dec}}(t)=\eta_{1} \Delta_{1} \sigma_{i}^{(1)}+\eta_{2} \Delta_{2} \sigma_{j}^{(2)} \quad(i, j=x, y, z)
$$

and the technique analyzed in the previous section can always be applied, more easily if $i=j$ in the above equation.

For a capacitive coupling (6.6), one finds two 2-dimensional Zeno subspaces when $i=j$ in Eq. (7.1). On the other hand, for a Josephson coupling (6.7), under the same conditions, there is only one 2-dimensional Zeno subspaces. As we have seen, inductive couplings (6.8) engender Zeno subspaces that are insensitive to noises along direction $x$ but are affected by noise along $y$.

In general, by suitably exploiting the symmetries of the system, one can always protect a given subspace in the strong coupling limit, but it is clearly impossible to protect a subspace from every kind of noise. In practice, one must first scrutinize the physical origin of the imperfections and 
then choose the suitable structure of the coupling in order to get the most robust subspaces.

The main physical problem to understand is whether it is realistic to consider a coupling that is strong enough, in the sense of Eq. (5.7), in order to generate the subspaces. In particular, in the case of inductive coupling, it is difficult to obtain a large value of the inductance $L$. However, some recent experiments using a Josephson junction device in a resonant cavity seem to pave the way towards this possibility. ${ }^{(13)}$

\section{ACKNOWLEDGMENTS}

This work is partially supported by the bilateral Italian-Japanese Projects II04C1AF4E on "Quantum Information Computation and Communication" of the Italian Ministry of Instruction, University and Research, and $15 \mathrm{C} 1$ on "Quantum Information and Computation" of the Italian Ministry for Foreign Affairs and by the Integrated Project "EuroSQIP" of the European Commission. T.Y. is supported by the Japanese Overseas Research Fellow Project of the Japanese Ministry of Education.

\section{REFERENCES}

1. A. O. Caldeira and A. J. Leggett, Ann. Phys. 149, 374 (1983).

2. D. Giulini, E. Joos, C. Kiefer, J. Kupsch, I.-O. Stamatescu, and H.-D. Zeh, Decoherence and the Appearance of a Classical World in Quantum Theory (Springer, Berlin, 1996); M. Namiki, S. Pascazio, and H. Nakazato, Decoherence and Quantum Measurements (World Scientific, Singapore, 1997).

3. U. Weiss, Quantum Dissipative Systems, 2nd ed. (World Scientific, Singapore, 1999); Y. Makhlin, G. Shon, and A. Shnirman, Rev. Mod. Phys. 73, 357 (2001).

4. M. A. Nielsen and I. L. Chuang, Quantum Computation and Quantum Information (Cambridge University Press, Cambridge, 2000); G. Benenti, G. Casati, and G. Strini, Principles of Quantum Computation and Information, Volume I: Basic Concepts (World Scientific, Singapore, 2004).

5. G. W. Ford, M. Kac, and P. Mazur, J. Math. Phys. 9, 504 (1965); V. B. Magalinskij, Zh. Eksp. Teor. Fiz. 36, 1942 (1959) [Sov. Phys. JEPT 91381 (1959)]; R. P. Feynman and F. L. Vernon, Ann. Phys. (N.Y.) 24, 118 (1963); P. Ullersma, Physica 32, 27 (1966); R. Zwanzig, J. Stat. Phys. 9, 215 (1973); A. O. Caldeira and A. J. Leggett, Phys. Rev. Lett. 46, 211 (1981); Physica A121, 587 (1983); Phys. Rev. A31 1059 (1985); V. Hakim and V. Ambegaokar, Phys. Rev. A32, 423 (1985); G. W. Ford and M. Kac, J. Stat. Phys. 46, 803 (1987); H. Grabert, P. Schramm, and G. L. Ingold, Phys. Rev. Lett. 58, 58 (1987); G. W. Ford, J. T. Lewis and R. F. O'Connell, Phys. Rev. A37, 4419 (1988).

6. Ph. Blanchard, G. Bolz, M. Cini, G. F. De Angelis, and M. Serva, J. Stat. Phys. 75, 749 (1994); M. Berry, in Fundamental Problems in Quantum Theory, D. M. 
Greenberger and A. Zeilinger eds. (Ann. N.Y. Ac. Sci. Vol. 755, New York, 1995), p. 303; H. Nakazato and S. Pascazio, J. Supercond. 12, 843 (1999).

7. C. W. Gardiner, and P. Zoller, Quantum Noise, 2nd ed. (Springer, Berlin, 2000).

8. V. Gorini, A. Kossakowski, and E. C. G. Sudarshan, J. Math. Phys. 17, 821 (1976); G. Lindblad, Comm. Math. Phys. 48, 119 (1976).

9. B. Misra and E. C. G. Sudarshan, J. Math. Phys. 18, 756 (1977); A. Beskow and J. Nilsson, Arkiv für Fysik 34, 561 (1967).

10. P. Facchi and S. Pascazio, Phys. Rev. Lett. 89080401 (2002); "Quantum Zeno subspaces and dynamical superselection rules," in Proceedings of the XXII Solvay Conference on Physics, I. Antoniou, V. A. Sadovnichy and H. Walther eds. (World Scientific, Singapore, 2003), p. 251. [quant-ph/0207030].

11. G. C. Wick, A. S. Wightman, and E. P. Wigner, Phys. Rev. 88, 101 (1952); Phys. Rev. D 1, 3267 (1970).

12. G. M. Palma, K. A. Suominen, and A. K. Ekert, Proc. R. Soc. Lond. A 452, 567 (1996); L. M. Duan and G. C. Guo, Phys. Rev. Lett. 79, 1953 (1997); P. Zanardi and M. Rasetti, Phys. Rev. Lett. 79, 3306 (1997).

13. Yu. Makhlin, G. Schön, and A. Shnirman, Nature 431, 138 (2004); I. Chiorescu et al., Nature 431, 159 (2004); A. Wallraff et al., Nature 431, 162 (2004); A. Blais et al., Phys. Rev. A 69, 062320 (2004); J. B. Majer et al., Phys. Rev. Lett. 94, 090501 (2005). 